Article

\title{
The Design of Optical Circuit-Analog Absorbers through Electrically Small Nanoparticles
}

\author{
Alessio Monti ${ }^{1, *(\mathbb{D})}$, Andrea Alù ${ }^{2}$, Alessandro Toscano ${ }^{3}$ and Filiberto Bilotti $^{3}$ \\ 1 Department of Engineering, Niccolò Cusano University, Via don Carlo Gnocchi 3, 00166 Rome, Italy \\ 2 CUNY Advanced Science Research Center, 85 St. Nicholas Terrace, New York, NY 10031, USA; \\ aalu@gc.cuny.edu \\ 3 Department of Engineering, Roma Tre University, Via Vito Volterra 62, 00146 Rome, Italy; \\ alessandro.toscano@uniroma3.it (A.T.); filiberto.bilotti@uniroma3.it (F.B.) \\ * Correspondence: alessio.monti@unicusano.it
}

Received: 5 February 2019; Accepted: 28 February 2019; Published: 6 March 2019

\begin{abstract}
In the last few years, the perfect absorption of light has become an important research topic due to its dramatic impact in photovoltaics, photodetectors, color filters and thermal emitters. While broadband optical absorption is relatively easy to achieve using bulky devices, today there is a strong need and interest in achieving the same effects by employing nanometric structures that are compatible with modern nanophotonic components. In this paper, we propose a general procedure to design broadband nanometer-scale absorbers working in the optical spectrum. The proposed devices, which can be considered an extension to optics of microwave circuit-analog absorbers, consist of several layers containing arrays of elongated nanoparticles, whose dimensions are engineered to control both the absorption level and the operational bandwidth. By combining a surface-impedance homogenization and an equivalent transmission-line formalism, we define a general analytical procedure that can be employed to achieve a final working design. As a relevant example, we show that the proposed approach allows designing an optical absorber exhibiting a $20 \%$ fractional bandwidth on a thickness of $\lambda / 4$ at the central frequency of operation. Full-wave results confirming the effectiveness of the analytical findings, as well as some considerations about the experimental realization of the proposed devices are provided.
\end{abstract}

Keywords: nanoparticles; optical losses; circuit-analog absorbers; surface dispersion effect; plasmonics

\section{Introduction}

The design of devices able to dissipate all the energy of an impinging electromagnetic wave into heat or other forms of energy, i.e., absorbers, has a long history that can be dated back to the World War II [1]. Nowadays, the use of RF and microwave absorbers is consolidated in many fields, such as electronic warfare and stealth technology [1], electromagnetic measurements [2], and antenna systems [3]. In the last few decades, there has been an increasing interest in extending similar effects to the visible spectrum [4-15]. Optical absorption, in fact, plays a key role in many relevant applications, including photovoltaics and thermophotovoltaics [16], photodetectors [17], color filters [18], and thermal light sources [19].

Recently, we have discussed the possibility to engineer the surface dispersion of small nanoparticles to achieve large optical absorption [20,21]. It is known that when the size of a plasmonic nanoparticle is smaller than the mean free path of electrons, its optical losses increase compared to the ones of the bulk material due to the additional scattering of electrons at the nanoparticle boundaries [22]. The arrangement of such small nanoparticles in an array configuration enables the design of ultrathin resistive sheets with a controlled amount of losses at a given frequency of the 
optical spectrum. Based on this concept, we have proposed an optical implementation of a Salisbury screen [20] and a possible variant featuring transparency outside its absorption bandwidth [21].

The aim of this contribution is to explore the suitability of nanoparticle arrays for the design of an advanced class of optical absorbers, i.e., the so-called circuit-analog (CA) absorbers. CA absorbers are popular at microwaves [23] for their larger absorption bandwidth compared to an equally thick Salisbury screen. The wider frequency response is obtained by replacing the resistive sheet with several reactive lossy layers that are able to compensate for the intrinsic reactance of the grounded dielectric around the resonance frequency of the structure. As we show in the following, arrays of plasmonic nanoparticles can be engineered to behave both as a resistive and a reactive layer and, thus, are suitable candidates for implementing CA absorbers in the visible spectrum.

We will show that, by exploiting the proposed approach, it is possible to design a three-layered CA absorber whose absorption bandwidth is 6 times bigger than the one of an optical Salisbury screen of equal thickness. Different from the conventional broadband solutions available in the market, the proposed absorbers are fully compatible with modern nanophotonic components and devices.

\section{Materials and Methods}

A CA absorber is a grounded structure consisting of $N$ reactive layers separated by dielectric spacers [1]. Its geometry for $N=3$ is sketched in Figure 1a. We assume that the absorber is illuminated by a transverse-magnetic (TM) plane wave whose wave vector $k_{i}$ forms an angle $\theta$ with the $z$-axis. The dielectric spacers have relative permittivity and permeability $\left(\varepsilon_{i}, \mu_{i}\right)$, whereas each reactive layer is characterized by a surface impedance $Z_{S}=R_{S}+j X_{s}$, whose real and imaginary parts account for the electromagnetic losses and the reactive behavior of the sheet, respectively.
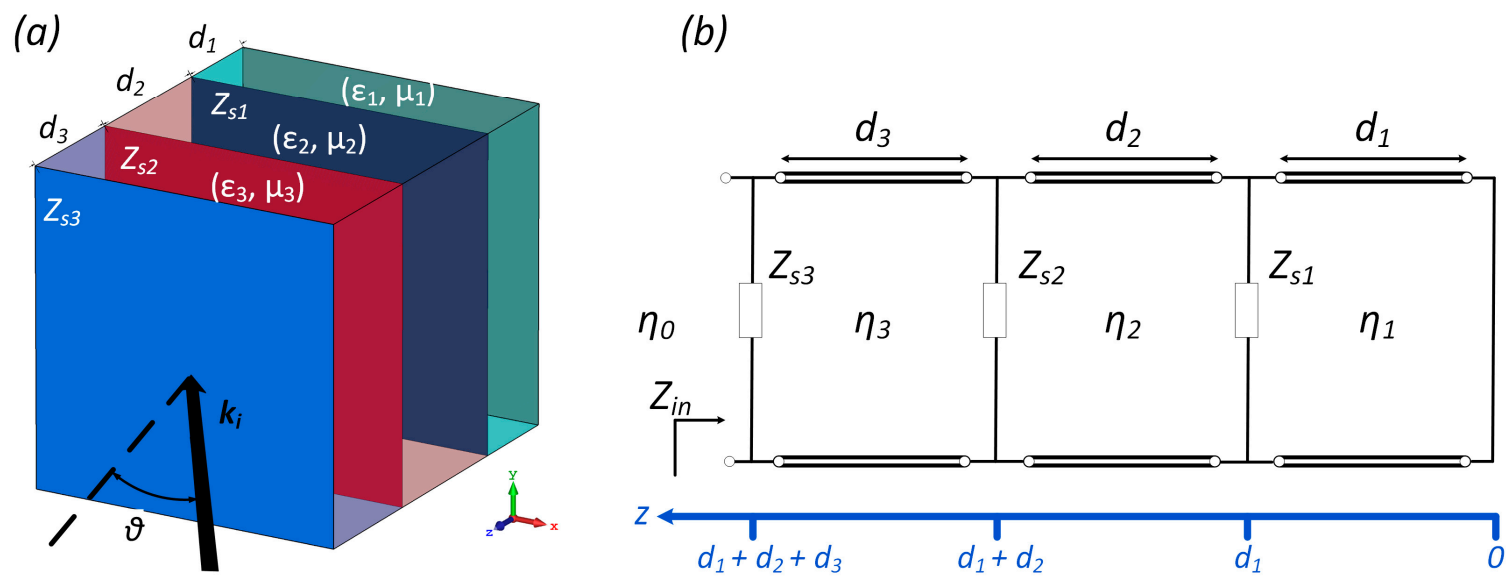

Figure 1. (a) A sketch of a three-layered circuit-analog absorber and (b) its equivalent circuit model.

The easiest strategy to design a CA absorber relies on the use of the Smith chart [23]. However, as it will be clear later, the strong frequency-dispersion of the nanoparticle-based optical resistive sheets limits the applicability of this simple approach in our scenario. An equivalent transmission-line representation of the device, such as the one shown in Figure 1b, is more convenient for our purposes. In this formalism, the $i$-th dielectric spacer is represented by a transmission line with length $d_{i}$ and characteristic impedance $\eta_{i}$, whereas each reactive layer is modeled through a shunt lumped impedance $Z_{s i}$.

The secondary constants of each transmission line segment, i.e., its characteristic impedance $\eta_{i}$ and propagation constant $\beta_{i}$, can be written in the following form:

$$
\left\{\begin{array}{c}
\eta_{i}=\frac{1}{\omega \varepsilon_{0} \varepsilon_{i}} \sqrt{\omega^{2} \mu_{0} \mu_{i} \varepsilon_{0} \varepsilon_{i}-k_{x}^{2}} \\
\beta_{i}=\sqrt{\omega^{2} \mu_{0} \mu_{i} \varepsilon_{0} \varepsilon_{i}-k_{x}^{2}}
\end{array}, i=1,2,3, \ldots, N,\right.
$$


where $\omega$ is the angular frequency and $k_{x}$ is the $x$-component of the free-space wave vector that is equal to

$$
k_{x}=\omega \sqrt{\mu_{0} \varepsilon_{0}} \sin (\theta) .
$$

For a normal-incident wave, the propagation constant of the $i$-th transmission line $\beta_{i}$ is equal to the dielectric wavenumber $k_{i}=\omega^{2} \mu_{0} \varepsilon_{0} \varepsilon_{i}$.

The design of a CA absorber involves the maximization of the absorption coefficient within a desired range of frequencies. Since CA absorbers are terminated on a ground plane and, thus, their transmission coefficient is zero, the maximization of the absorption corresponds to the minimization of the input reflection coefficient. By relying on the model shown in Figure 1b, the input reflection coefficient can be easily calculated using the conventional transmission line formulas. In particular, the input impedance of a load $Z_{L}$ at a given $z$ can be calculated as [24]:

$$
Z(z)=\eta_{i} \frac{Z_{L}+j \eta_{i} \tan \left(\beta_{i} z\right)}{\eta_{i}+j Z_{L} \tan \left(\beta_{i} z\right)} .
$$

Using Equation (3), it is easy to calculate the line impedance before the first reactive layer:

$$
Z\left(d_{1}^{-}\right)=j \eta_{1} \tan \left(\beta_{1} d_{1}\right) .
$$

Such an impedance is shunt-connected to the surface impedance of the reactive layer and, thus, the overall impedance at $z=d_{1}$ is equal to

$$
Z\left(d_{1}\right)=\left(\frac{1}{Z_{s 1}}+\frac{1}{Z\left(d_{1}^{-}\right)}\right)^{-1}
$$

These simple analytical steps can be recursively applied to the two subsequent dielectric spacers and shunt layers in order to calculate the overall input impedance $Z_{\text {in }}$ at $z=d_{1}+d_{2}+d_{3}$. Once $Z_{\text {in }}$ is known, the input reflection coefficient can be expressed as follows:

$$
\Gamma(\omega, \vartheta)=\frac{Z_{\text {in }}(\omega, \vartheta)-\eta_{0}}{Z_{\text {in }}(\omega, \vartheta)+\eta_{0}}
$$

where $\eta_{0}=377 \Omega$ is the free-space impedance. The above procedure can be easily extended to a CA absorber made by an arbitrary number of layers $N$ or even to non-homogenous dielectric layers [25,26].

Before moving forward, it is necessary to specify how to implement the reactive layers required by a CA absorber at optical frequencies. At microwaves, such layers can be realized by patterning a lossy material $[27,28]$ or by adding lossy lumped elements to a reactive surface [29]. As to the optical frequencies, we have recently proven that a resistive sheet can be realized using an array of ellipsoidal nanoparticles that are smaller than the mean free path of electrons in the constituent plasmonic material [20]. However, we have also shown that, by properly tuning the eccentricity of the nanoparticles and their separation distance, it is possible to add a capacitive or inductive behavior to the lossy array [30-32].

In this paper, thus, the reactive layers are implemented through a square array of nanoparticles, such as the one shown in Figure 2a. The nanoparticles are prolate spheroids with $r_{x}=r_{z}$ and they are made of silver. Their permittivity is expressed through the following size-corrected dielectric function [33]:

$$
\varepsilon_{A g}\left(\omega, r_{i}\right)=1-\frac{\omega_{p}^{2}}{\omega\left(\omega-j\left(\gamma+A \frac{v_{F}}{r_{i}}\right)\right)}+\frac{f \omega_{L}^{2}}{\omega_{L}^{2}-\omega^{2}+j \Gamma_{L} \omega}, \quad i=x, y, z .
$$


The values of the parameters appearing in Equation (7) are $\omega_{p}=9.17 \mathrm{eV}, \omega_{L}=5.27 \mathrm{eV}, f=2.2$, $\Gamma_{L}=1.14 \mathrm{eV}, \gamma=0.0023 \times \omega_{p}, v_{F}=1.39 \times 10^{6} \mathrm{~m} / \mathrm{s}, A=0.6$. It is important to underline that, compared to the conventional Drude model of silver, Equation (7) contains an additional loss contribution that is inversely proportional to the radius of the nanoparticle. This term accounts for the surface dispersion that allows tuning the surface resistance of the nanoparticle array.

By assuming that the size of the nanoparticles and their separation distance are significantly smaller than the operative wavelength, the structure in Figure $2 \mathrm{a}$ can be homogenized through effective bulk or surface parameters. There exist several alternative methods for describing the optical behavior of the structure shown in Figure 2a, such as volumetric homogenization [34,35] and equivalent conductivity methods [36]. However, the approach based on the definition of an effective surface impedance is the most suitable for the design of ultra-thin layers exhibiting both resistive and reactive behaviors. According to it, when illuminated by an external normally-impinging plane wave with the electric field parallel to the major axis of the ellipsoids, the structure shown in Figure 2a behaves as a lossy reactive layer, whose surface impedance is [20]

$$
Z_{s}^{y}=R_{s}^{y}+j X_{s}^{y}=-j \frac{a^{2}}{k}\left(\alpha_{y}^{-1}-\beta\right),
$$

where $a$ is the separation distance among nanoparticles, $\alpha_{y}$ the $y$-component of the nanoparticle polarizability tensor [22], $k=k_{0} \sqrt{\varepsilon_{h}}$ the wavenumber in the hosting material, and, finally, $\beta$ is the interaction constant among nanoparticles. An approximate expression for $\beta$ for the case of normal incidence is available, for instance, in Reference [37].

(a)

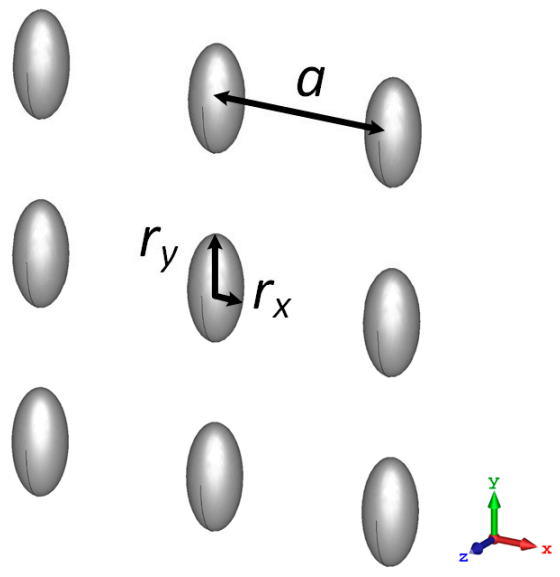

(b)

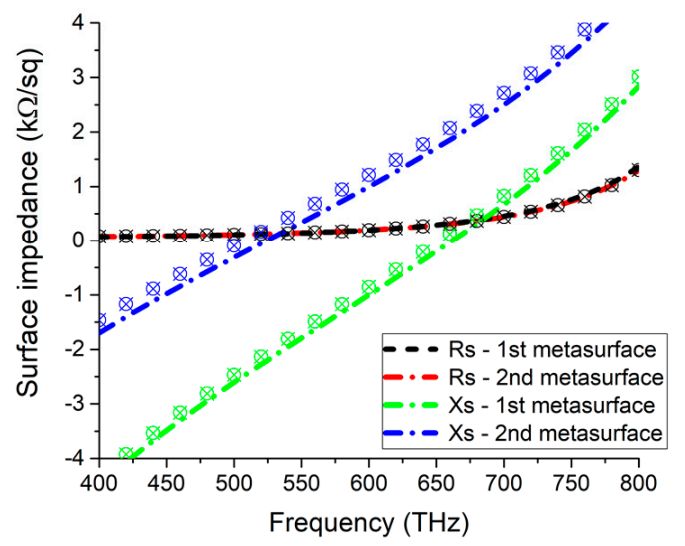

Figure 2. (a) The optical metasurface made by an array of ellipsoidal plasmonic nanoparticles arranged in a square lattice; (b) the complex surface impedance of two different optical metasurfaces designed to have the same surface resistance $\left(R_{S}=200 \Omega\right)$ and opposite reactive behaviors (capacitive and inductive). The ticks represent the results of full-wave simulations.

By exploiting the Equation (8), we have analytically designed two different metasurfaces exhibiting the same surface resistance $\left(R_{S}=200 \Omega\right)$ but opposite surface reactance $\left(X_{S 1}=-1000 \Omega / \mathrm{sq}\right.$ and $X_{s 2}=1000 \Omega / \mathrm{sq}$, respectively) at $f=600 \mathrm{THz}$. Their dimensions are as follows: (1st) $r_{y}=16 \mathrm{~nm}$, $r_{x}=6 \mathrm{~nm}, a=55 \mathrm{~nm}$; (2nd) $r_{y}=22 \mathrm{~nm}, r_{x}=5 \mathrm{~nm}, a=55 \mathrm{~nm}$. The frequency behavior of the complex surface impedance for these arrays of nanoparticles is shown in Figure $2 b$. The ticks represent the numerical values that have been retrieved through full-wave simulations. As can be appreciated, such a configuration allows achieving a non-resonant lossy behavior at a desired frequency of the visible spectrum. Specifically, the surface reactance is mainly related to the nanoparticle eccentricity, whereas the surface resistance can be tuned by acting on the absolute size of the nanoparticles [20]. Thus, by engineering the major and minor axes of the nanoparticles, it is possible to obtain an optical 
metasurface with a desired amount of losses and either a capacitive or an inductive behavior. It is interesting to observe that, differently from their microwave versions, the surface impedance of an optical metasurface based on nanoparticles is strongly frequency-dispersive, even in its real part. This implies that the maximum achievable bandwidth of a nanoparticles-based optical absorber is unavoidably lower than the one of a corresponding CA absorber working at microwave frequencies.

By introducing Equation (8) into the reflection coefficient (6), we can find an analytical expression of the reflection coefficient that depends on the dimensions of the different nanoparticle arrays composing the CA absorber. As we will show in the next Section, this expression can be analytically minimized within the desired range of frequencies of the optical spectrum with respect to the geometrical parameters of the structure.

Before concluding this Section, we would like to underline that, given the geometrical and electromagnetic anisotropy of the ellipsoids composing the optical metasurfaces, their response is intrinsically polarization-sensitive, i.e., they exhibit different values of surface resistance and reactance depending on the orientation of the incident electric field. While we have shown that it is possible to make these structures quasi-isotropic by properly arranging different replicas of the nanoparticles [20], we also remark that such a feature can be considered an advantage in several applications, such as the design of optical polarizers or polarization-sensitive devices.

\section{Results}

In the previous Section, we discussed an analytical model able to describe the behavior of a CA absorber whose lossy reactive layers are implemented through plasmonic nanoparticle arrays. Here, we show how this model can be exploited for designing a wideband optical absorber.

The design of a CA absorber is based on the numerical minimization of the discrete frequency integral of the reflection coefficient (6) with respect to the design parameters, i.e., the dimensions of the two arrays of nanoparticles and the thicknesses of the dielectric spacers:

$$
r_{y}^{1}, r_{x}^{1}, r_{y}^{2}, r_{y}^{2}, d_{1}, d_{2}\left\{\sum_{f=f_{\min }}^{f=f_{\max }} \Gamma(f, \vartheta)\right\}
$$

However, to ensure the physical realizability of the absorber, some design constraints must be imposed to Equation (9): first, the separation distance among the nanoparticles must be greater than twice the major axis, i.e., $a>2 r_{y}$; then, we may want to take into account the limitations of the most common nanofabrication facilities and force a minimum nanoparticle size, i.e., $r_{x}^{1}, r_{x}^{2}>r_{\min }$. Moreover, in order to minimize the coupling between metasurfaces that may affect their effective surface impedance, we need to impose that the two nanoparticle arrays have a minimum separation, i.e., $d_{1}, d_{2}>\lambda / 15, \lambda$ being the wavelength inside the dielectric at the central frequency of operation. Finally, we can impose that the overall thickness of the CA absorber is less than or equal to the one of a conventional Salisbury screen, i.e., $d_{1}+d_{2}<\lambda / 4$.

We start by considering the case of a CA absorber with $N=2$, whose structure is shown in Figure 3a. The ideal infinitely-thin metallic ground plane shown in Figure 1a is now replaced by a thick enough layer of silver $(t=50 \mathrm{~nm})$ that minimizes the transmission of light beyond the structure. We assume that the two nanoparticle arrays are placed on a silica layer, which is a non-magnetic material commonly used as a substrate for nanoparticles $\left(\varepsilon_{d}=2.1\right)$. It is worth noticing that the choice of the dielectric substrate affects both the thickness of the absorber (equal to $\lambda / 4$ at the central frequency of operation) and the optimal size of the nanoparticles returned by the optimization process. Assuming a normal incidence, the reflection coefficient (6) reads as

$$
\frac{\tan \left(d_{1} k_{d}\right)\left[\tan \left(d_{2} k_{d}\right)\left(Z_{s}^{1} \eta_{0}^{2} \omega \varepsilon_{0}-Z_{\times} k_{0} \varepsilon_{d}-\eta_{0}^{2} k_{0}\right)+j Z_{s}^{2} \eta_{0} \sqrt{\varepsilon_{d}}\left(k_{0}-Z_{s}^{1} \omega \varepsilon_{0}\right)\right]+j \eta_{0} \sqrt{\varepsilon_{d}} \tan \left(d_{2} k_{d}\right)\left[k_{0} Z_{+}-Z_{\times} \omega \varepsilon_{0}\right]+Z_{\times} k_{0} \varepsilon_{d}}{\tan \left(d_{1} k_{d}\right)\left[\tan \left(d_{2} k_{d}\right)\left(Z_{s}^{1} \eta_{0}^{2} \omega \varepsilon_{0}+Z_{\times} k_{0} \varepsilon_{d}+\eta_{0}^{2} k_{0}\right)-j Z_{s}^{2} \eta_{0} \sqrt{\varepsilon_{d}}\left(k_{0}+Z_{s}^{1} \omega \varepsilon_{0}\right)\right]-j \eta_{0} \sqrt{\varepsilon_{d}} \tan \left(d_{2} k_{d}\right)\left[k_{0} Z_{+}+Z_{\times} \omega \varepsilon_{0}\right]-Z_{\times} k_{0} \varepsilon_{d}}
$$


where $k_{d}$ is the wavenumber in silica, $Z_{s}{ }^{1}$ and $Z_{s}{ }^{2}$ are the complex surface impedances of the first and the second reactive layer, respectively, and $Z_{x}=Z_{s}^{1} Z_{s}^{2}, Z_{+}=Z_{s}^{1}+Z_{s}^{2}$. It is important to underline that the first optical reactive layer is completely immersed in silica and, thus, the permittivity of the host medium to be used in (8) is the one of silica. Conversely, as it should be clear from Figure 3a, the second reactive layer is placed between the dielectric and the vacuum. Thus, an average permittivity between these two dielectrics must be used as $\varepsilon_{h}$.

The discrete frequency integral of the reflection coefficient (10) has been minimized under the described design constraints by assuming a central frequency $f=600 \mathrm{THz}$ and as minimum and maximum frequencies $f_{\min }=550 \mathrm{THz}, f_{\max }=650 \mathrm{THz}$, respectively. This leads to the following optimal parameters: $a_{1}=a_{2}=29 \mathrm{~nm}, r_{y}{ }^{1}=r_{y}{ }^{2}=8 \mathrm{~nm}, r_{x}{ }^{1}=r_{x}{ }^{1}=4 \mathrm{~nm}, d_{1}=55 \mathrm{~nm}, d_{2}=30 \mathrm{~nm}$. It is important noticing that, even though the dimensions of the two nanoparticle arrays are the same, their surface reactance (shown in Figure $3 b$ ) is different because of the different effective permittivity of their host material. In Figure 3b, we also show the input reactance of the absorber evaluated at $z=d_{1}^{-}$ and $z=d_{2}^{-}$, i.e., before the first and the second reactive layer, respectively. From this comparison, it should be clear that the optimization has led to reactive layers, whose surface reactance is engineered to compensate for the intrinsic dispersion of the dielectrics.
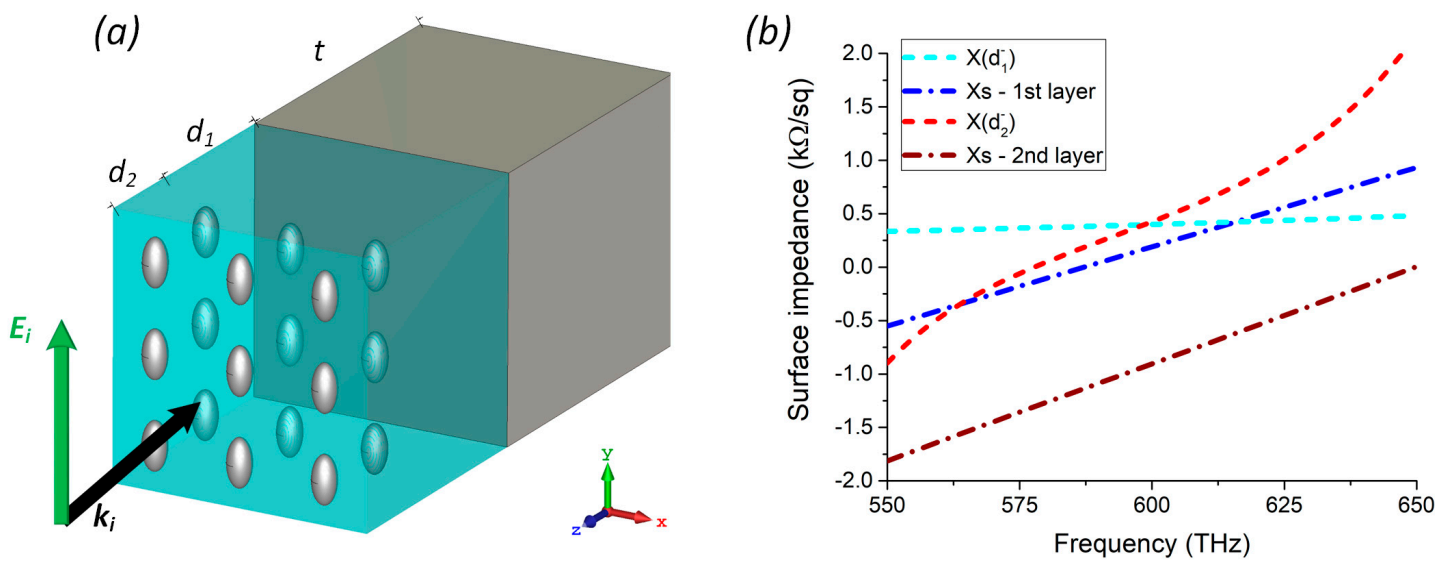

Figure 3. (a) The two-layered optical circuit-analogue (CA) absorber. The ground plane is replaced by a thick enough layer of silver; (b) the surface reactance of the two metasurfaces required to maximize the absorption within the frequencies range 550-650 THz. The surface reactances of the metasurfaces are compared with the input reactance of the absorber evaluated before the first and the second layer.

In Figure $4 \mathrm{a}$, we show the analytical real and imaginary parts of the overall input impedance $Z_{\text {in }}$ at $z=d_{1}+d_{2}$. The input impedance is compared to the one of a single-layer optical Salisbury screen, whose resistive sheet is resonant and impedance-matched with the vacuum at $600 \mathrm{THz}$ [20]. As expected, the input reactance of the Salisbury screen is equal to zero only at a single frequency and increases quickly as we move away from the resonance. Conversely, the CA-absorber exhibits an input reactance that is close to zero within a wide range around the design frequency. This is confirmed by the results shown in Figure $4 b$, where the absorbance of the two absorbers is compared. The CA absorber has a significantly broader absorption bandwidth due to the optimized reactive behavior of the lossy layers. Specifically, assuming an absorbance threshold of 0.9 , the fractional bandwidth of the CA absorber is $13.2 \%$, almost 4 times bigger than the one of the optical Salisbury screen. We stress that this comparison has been carried out by considering two absorbers with the same overall thickness.

The analytical absorbance of the CA absorber is compared to the one obtained through full-wave simulations carried out on a unit-cell of the CA absorber. As can be appreciated, there is a good agreement between the numerical and theoretical results, confirming the effectiveness of the method discussed in the previous Section. The agreement slightly deteriorates at higher frequencies mainly due to the progressive violation of the assumptions made to derive Equation (8) and to the non-perfect reflection of the backing silver layer. A possibility to reduce the transmittance of the absorber without 
increasing further its overall thickness could rely on the use of ultrathin optical mirrors based on dielectric nanoparticles [38]. This would also make the design of broadband absorbers that are transparent outside their absorption bandwidth possible (similar to the narrowband design we have discussed in Reference [21]).

(a)

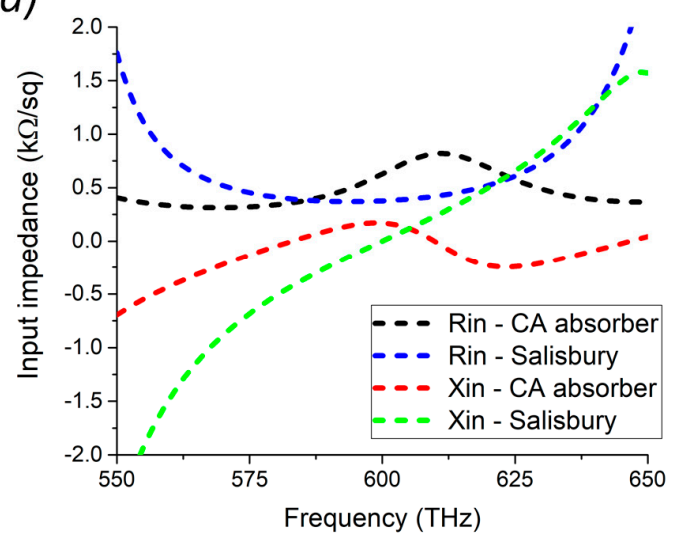

(b)

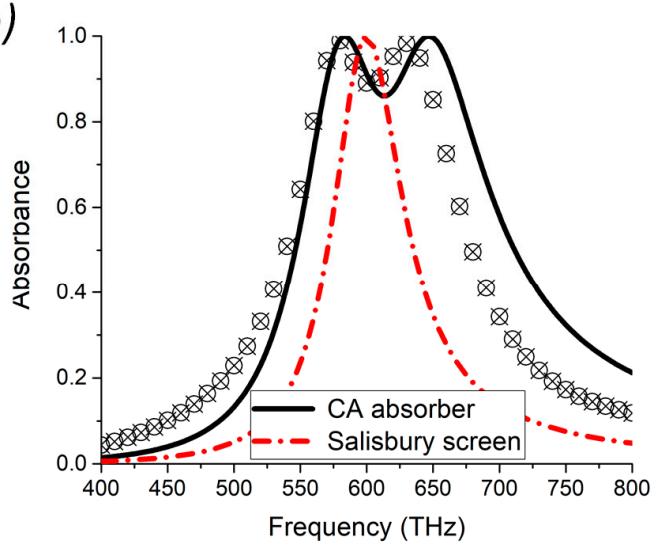

Figure 4. (a) The input impedance of the designed CA absorber and of an equivalent Salisbury screen with the same thickness. (b) The analytical and numerical absorbance (absolute value) of the designed CA absorber and of an equivalent Salisbury screen with the same thickness. Ticks represent the results of full-wave simulations.

We consider now the case of a CA absorber made by three layers $(N=3)$. We apply the minimization procedure described above with the same design constraints and, in particular, by fixing the maximum thickness of the absorber equal to the one of the Salisbury screen. After a quick numerical optimization, we get the following optimum geometrical values: $a_{1}=a_{2}=a_{3}=32 \mathrm{~nm}$, $r_{y}{ }^{1}=9 \mathrm{~nm}, r_{x}{ }^{1}=5 \mathrm{~nm}, r_{y}^{2}=10 \mathrm{~nm}, r_{x}{ }^{2}=4 \mathrm{~nm}, r_{y}{ }^{3}=8 \mathrm{~nm}, r_{x}{ }^{3}=4 \mathrm{~nm}, d_{1}=40 \mathrm{~nm}, d_{2}=25 \mathrm{~nm}$, $d_{3}=25 \mathrm{~nm}$. The reflectance, absorbance and transmittance of this absorber, obtained through full-wave simulations, are shown in Figure 5a. As can be appreciated, the absorbance stays above 0.9 within the frequency range $540-670 \mathrm{THz}$, resulting in a fractional bandwidth bigger than $20 \%$ with a thickness of only $\lambda / 4$ at the center frequency of the absorption band. In Figure $5 b$, we report the value of the absorbance at three different frequencies as the impinging angle $\theta$ changes. As can be appreciated, for all the considered frequencies, the absorbance is larger than 0.9 for a wide range of incidence angles, up to $50^{\circ}$.

(a)

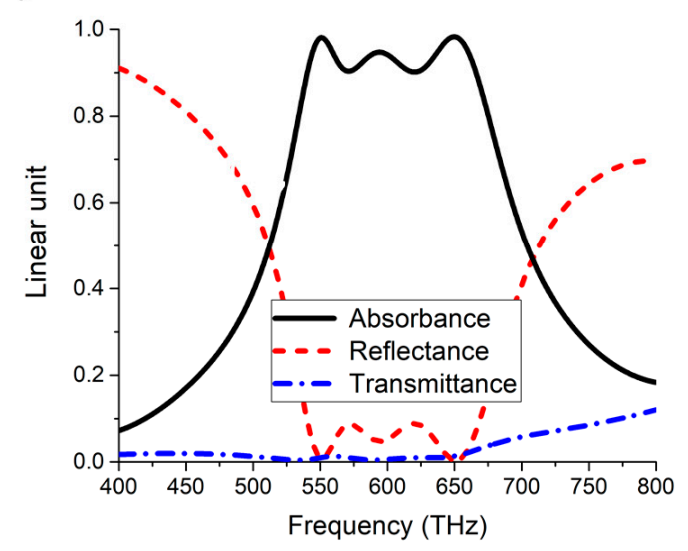

(b)

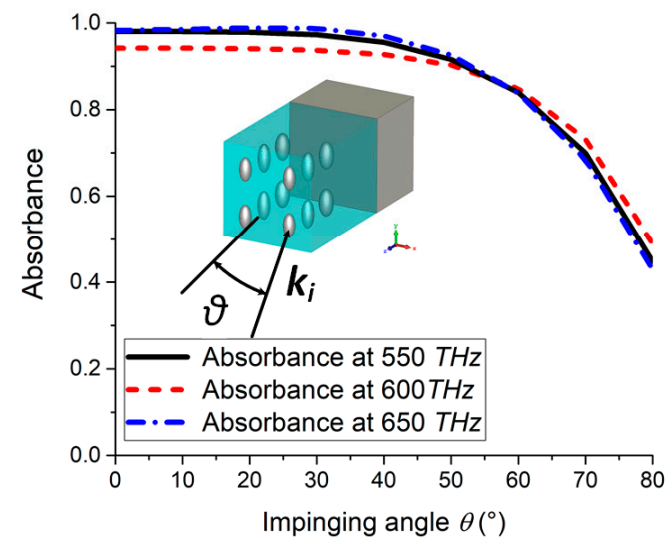

Figure 5. (a) The absorbance, reflectance and transmittance (absolute value) of a three-layered CA optical absorber obtained through full-wave simulations; (b) the value of the absorbance (absolute value) at three different frequencies as the impinging angle changes. 
An interesting comparison between the Salisbury screen and the CA absorbers can be carried out by exploiting the metric defined by the Rozanov's limit [39]. Accordingly, the minimum thickness of a metal-backed absorber needed to achieve a reflectance $R(\lambda)$ is equal to

$$
d_{\min }=\frac{\int_{\lambda} \ln |R(\lambda)| d f}{2 \pi^{2}} .
$$

Being theoretical bound, this parameter can be used to quantify univocally the performances of any grounded absorber. In particular, in our cases, the ratio between the actual thickness of the absorber and its theoretical minimum is equal to $8 \%, 21 \%$ and $28 \%$, for the Salisbury screen and for the 2- and 3-layered CA absorber, respectively. Though the theoretical limit is still distant, mainly due to the strong dispersion of the nanoparticles-based optical metasurfaces, the improvement with respect to the Salisbury screen is significant.

Before concluding this Section, we would like to stress that the approach discussed in this manuscript to increase the absorption bandwidth of a Salisbury screen is inherently different from the one employed in the Jaumann absorber [1]. The broadband absorption of a Jaumann screen, in fact, is achieved by stacking purely-resistive layers separated by different quarter-wavelength-thick dielectric layers. This approach significantly increases the overall thickness of the whole device. Conversely, CA absorbers exploit the additional degree of freedom provided by the reactive behavior of the lossy layers and allows for achieving wide absorption bandwidth without increasing the thickness of the Salisbury screen.

\section{Alternative Layout and Sensitivity Analysis}

The aim of this Section is to provide some interesting considerations about the geometry of the proposed absorbers in view of their possible experimental realization. For this purpose, (i) we propose an alternative geometry that may facilitate the experimental realization of the proposed CA-absorbers; (ii) we investigate its robustness towards some common inaccuracies that may arise during the nanofabrication process of the optical metasurfaces.

The first aspect we would like to discuss is the geometry of the nanoparticles composing the optical metasurfaces. In fact, the CA absorbers described in the previous Section require plasmonic ellipsoids, whose experimental realization, though possible, is not straightforward. However, as it should be clear from the discussion in Section 2, the basic design principle of these devices relies on the control of the eccentricity and of the absolute size of nanoparticles. In other terms, their exact shape is not fundamental as far as it remains elongated. Therefore, we can replace the ellipsoids shown in Figure 2a with nanocylinders that are much easier to be experimentally realized $[40,41]$. Unfortunately, a closed-form expression of the polarizability of finite-size cylinders is not known and, thus, we cannot extend Equation (8) to the case of an array of cylinders.

To overcome this issue, we need to numerically optimize the optical metasurfaces based on nanorods. For this purpose, in Figure 6a we show the comparison between the complex surface impedance exhibited by the two optical metasurfaces made by ellipsoids and cylinders with the same linear dimensions embedded in a silica matrix. The values of the major and minor axes used for this example are the ones of the second layer of the three-layered CA absorber discussed in the previous section. As can be appreciated, the surface reactance is affected by the change of the shape, consistent with the discussion about their static polarizabilities available in [42]. We need, thus, to re-optimize the size of the nanoparticles. Since the surface reactance mainly depends on the eccentricity, we may conveniently limit our optimization to the major axis of the cylinders. After some retrieval based on full-wave simulations, we get that a $30 \%$ reduction of the major axes allows for the restoration of the original surface reactance exhibited by the three layers. Please note that, in principle, these geometrical changes also affect the surface resistance of the metasurface. However, since we have only reduced the major axis without changing the minor ones, the variation of the surface resistance is negligible 
and does not significantly affect the performance of the absorber. This is confirmed by the full-wave absorbance of the nanocylinders-based CA absorber shown in Figure 6b (black line).

The second important aspect we would like to investigate is the robustness of the device performances towards common manufacturing inaccuracies that may arise during the fabrication process. To this purpose, we have considered a square sample of the three-layered CA absorber made by $4 \times 4$ unit-cells. Each unit-cell is composed of three nanocylinders stacked along the $z$-axis. In order to introduce a realistic perturbation, we have randomly divided the 48 cylinders into 6 groups. The elements of the first two groups have been translated along the $x$-axis by a quantity $\pm \Delta$, respectively. Similarly, the elements of the third and fourth group have been moved along the $y$-axis by a quantity $\pm \Delta$, respectively. Finally, the elements of the last two groups have been translated both along the $x$ and the $y$-axis by quantity $\pm \Delta$, respectively. The full-wave results for different values of $\Delta$ are shown in Figure $6 \mathrm{~b}$. As can be appreciated, the response of the absorber is almost insensitive to the exact positions of the nanoparticles in the arrays. This is an expected result since, as described in Section 2, the surface impedance of an optical metasurface is an average effect that does not depend on the exact position of the nanoparticles in the lattice. It is worth noticing that the proposed perturbation analysis is quite conservative since we have assumed that all nanoparticles are moved with respect to their original position of a quantity that is comparable to the length of their major axis.

(a)

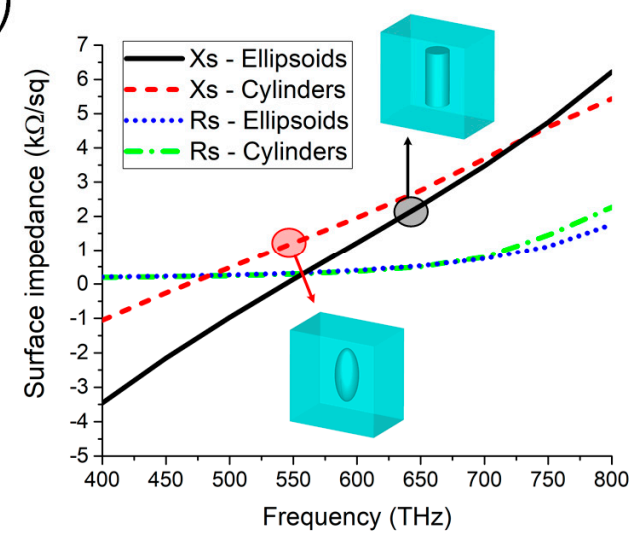

(b)

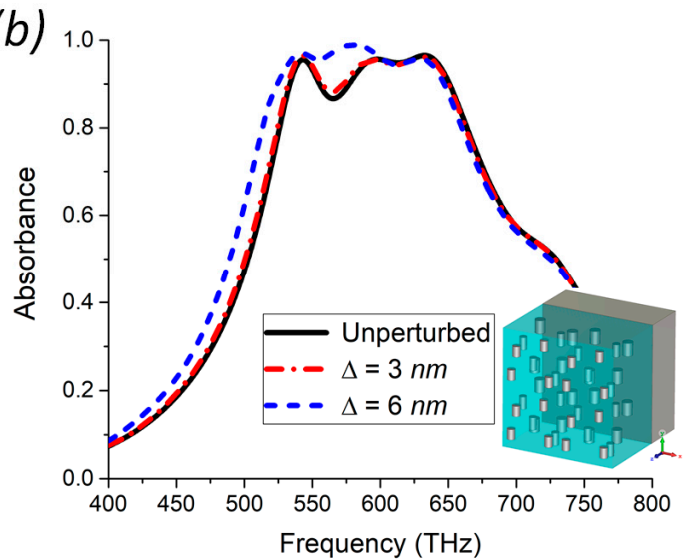

Figure 6. (a) The complex surface impedance exhibited by two arrays of nanoellipsoids and nanocylinders with the same size; (b) the absorbance of the three-layered CA absorber made by nanocylinders for different values of the perturbation parameter $\Delta$. In the inset, the equivalent absorber structure for $\Delta=6 \mathrm{~nm}$ is shown.

\section{Conclusions}

In this paper, we have described a strategy to design broadband circuit-analog absorbers operating at optical frequencies. The proposed absorbers consist of different layers made of arrays of plasmonic nanoparticles, whose size and eccentricity are optimized to achieve perfect optical absorption in a broad frequency range. Specifically, the absorption is achieved by engineering the surface dispersion occurring in electrically small nanoparticles, whereas the broadband response is due to the reactive behavior of the layers that compensate for the intrinsic dispersion of the grounded dielectric. An analytical procedure has been described and validated through full-wave simulations. In particular, we have shown that it is possible to increase (by a factor of 6) the absorption bandwidth of an optical Salisbury screen by designing a three-layer CA absorber with the same thickness. Finally, in view of a possible experimental realization, we have proposed an alternative layout made of nanocylinders and investigated its robustness towards common manufacturing inaccuracies.

Author Contributions: A.M., A.A., A.T. and F.B. conceived the idea; A.M. carried out the analytical derivations and numerical examples; A.M., A.A., A.T. and F.B. analyzed and interpreted the results and contributed to the writing of the manuscript. 
Funding: This research received no external funding.

Conflicts of Interest: The authors declare no conflict of interest.

\section{References}

1. Knott, E.F. Radar Cross Section, 2nd ed.; SciTech Publishing: Raleigh, NC, USA, 2004.

2. Emerson, W. Electromagnetic wave absorbers and anechoic chambers through the years. IEEE Trans. Antennas Propag. 1973, 21, 484-490. [CrossRef]

3. Beeharry, T.; Yahiaoui, R.; Selemani, K.; Ouslimani, H.H. A dual layer broadband radar absorber to minimize electromagnetic interference in radomes. Sci. Rep. 2018, 8, 382. [CrossRef] [PubMed]

4. Liu, N.; Mesch, M.; Weiss, T.; Hentschel, M.; Giessen, H. Infrared perfect absorber and its application as plasmonic sensor. Nano Lett. 2010, 10, 2342-2348. [CrossRef] [PubMed]

5. Aydin, K.; Ferry, V.E.; Briggs, R.M.; Atwater, H.A. Broadband polarization-independent resonant light absorption using ultrathin plasmonic super absorbers. Nat. Commun. 2011, 2, 517. [CrossRef] [PubMed]

6. Wu, C.; Neuner, B.; Shvets, G.; John, J.; Milder, A.; Zollars, B.; Savoy, S. Large-area wide-angle spectrally selective plasmonic absorber. Phys. Rev. B 2011, 84, 075102. [CrossRef]

7. Alici, K.B.; Turhan, A.B.; Soukoulis, C.M.; Ozbay, E. Optically thin composite resonant absorber at the near-infrared band: A polarization independent and spectrally broadband configuration. Opt. Express 2011, 19, 14260-14267. [CrossRef] [PubMed]

8. Moreau, A.; Ciracì, C.; Mock, J.J.; Hill, R.T.; Wang, Q.; Wiley, B.J.; Chilkoti, A.; Smith, D.R. Controlled-reflectance surfaces with film-coupled colloidal nanoantennas. Nature 2012, 492, 86-89. [CrossRef] [PubMed]

9. Chang, Y.-C.; Kildishev, A.V.; Narimanov, E.E.; Norris, T.B. Metasurface perfect absorber based on guided resonance of a photonic hypercrystal. Phys. Rev. B 2016, 94, 155430. [CrossRef]

10. Khan, A.D.; Amin, M. Tunable Salisbury screen absorber using square lattice of plasmonic nanodisk. Plasmonics 2016, 12, 257-262. [CrossRef]

11. Hao, J.; Wang, J.; Liu, X.; Padilla, W.J.; Zhou, L.; Qiu, M. High performance optical absorber based on a plasmonic metamaterial. Appl. Phys. Lett. 2010, 96, 251104. [CrossRef]

12. Ra'di, T.; Asadchy, V.S.; Kosulnikov, S.U.; Omelyanovich, M.M.; Morits, D.; Osipov, A.V.; Simovski, C.R.; Tretyakov, S.A. Full light absorption in single arrays of spherical nanoparticles. ACS Photonics 2015, 2, 653-660. [CrossRef]

13. Akselrod, G.B.; Huang, J.; Hoang, T.B.; Bowen, P.T.; Su, L.; Smith, D.R.; Mikkelsen, M.H. Large-area metasurface perfect absorbers from visible to near-infrared. Adv. Mater. 2015, 27, 8028. [CrossRef] [PubMed]

14. Bowen, P.T.; Baron, A.; Smith, D.R. Theory of patch-antenna metamaterial perfect absorbers. Phys. Rev. A 2016, 93, 063849. [CrossRef]

15. Jiang, X.; Shao, L.; Zhang, S.X.; Yi, X.; Wiersig, J.; Wang, L.; Gong, Q.; Lončar, M.; Yang, L.; Xiao, Y.-F. Chaos-assisted broadband momentum transformation in optical microresonators. Science 2017, 358, $344-347$. [CrossRef] [PubMed]

16. Atwater, H.A.; Polman, A. Plasmonics for improved photovoltaic devices. Nat. Mater. 2010, 9, $205-213$. [CrossRef] [PubMed]

17. Mokkapati, S.; Saxena, D.; Tan, H.H.; Jagadish, C. Optical design of nanowire absorbers for wavelength selective photodetectors. Sci. Rep. 2015, 5, 15339. [CrossRef] [PubMed]

18. Mirshafieyan, S.S.; Gregory, D.A. Electrically tunable perfect light absorbers as color filters and modulators. Sci. Rep. 2018, 8, 2635. [CrossRef] [PubMed]

19. Wang, Z.; Tong, Z.; Ye, Q.; Hu, H.; Nie, X.; Yan, C.; Shang, W.; Song, C.; Wu, J.; Wang, J.; et al. Dynamic tuning of optical absorbers for accelerated solar-thermal energy storage. Nat. Commun. 2018, 8, 1478. [CrossRef] [PubMed]

20. Monti, A.; Toscano, A.; Bilotti, F. Exploiting the surface dispersion of nanoparticles to design optical-resistive sheets and Salisbury absorbers. Opt. Lett. 2016, 241, 3383-3386. [CrossRef] [PubMed]

21. Monti, A.; Alù, A.; Toscano, A.; Bilotti, F. Narrowband transparent absorbers based on ellipsoidal nanoparticles. Appl. Opt. 2017, 56, 7533-7538. [CrossRef] [PubMed]

22. Noguez, C. Optical properties of isolated and supported metal nanoparticles. Opt. Mater. 2005, $27,1204$. [CrossRef] 
23. Munk, B.A.; Munk, P.; Pryor, J. On designing Jaumann and circuit analog absorbers (CA absorbers) for oblique angle of incidence. IEEE Trans. Antennas Propag. 2007, 55, 186-193. [CrossRef]

24. Pozar, D. Microwave Engineering; Wiley: Hoboken, NJ, USA, 2012.

25. Vegni, L.; Toscano, A. Full-wave analysis of planar stratified media with inhomogeneous layers. IEEE Trans. Antennas Propag. 2000, 48, 631-633. [CrossRef]

26. Urbani, F.; Vegni, L.; Toscano, A. Inhomogeneous layered planar structures: An analysis of reflection coefficient. IEEE Trans. Magn. 1998, 35, 2771-2774. [CrossRef]

27. Shang, Y.; Shen, Z.; Xiao, S. On the design of single-layer circuit analog absorber using double-square-loop array. IEEE Trans. Antennas Propag. 2013, 61, 6022-6029. [CrossRef]

28. Landy, N.I.; Sajuyigbe, S.; Mock, J.J.; Smith, D.R.; Padilla, W.J. Perfect metamaterial absorber. Phys. Rev. Lett. 2008, 100, 207402. [CrossRef] [PubMed]

29. Chen, J.; Shang, Y.; Liao, C. Double-layer circuit analog absorbers based on resistor-loaded square-loop arrays. IEEE Antennas Wirel. Propag. Lett. 2018, 17, 591-595. [CrossRef]

30. Monti, A.; Alù, A.; Toscano, A.; Bilotti, F. Optical scattering cancellation through arrays of plasmonic nanoparticles: A review. Photonics 2016, 2, 540-552. [CrossRef]

31. Monti, A.; Toscano, A.; Bilotti, F. Analysis of the scattering and absorption properties of ellipsoidal nanoparticle arrays for the design of full-color transparent screens. J. Appl. Phys. 2017, 121, 243106. [CrossRef]

32. Monti, A.; Alù, A.; Toscano, A.; Bilotti, F. Metasurface-based anti-reflection coatings at optical frequencies. J. Opt. 2018, 20, 055001. [CrossRef]

33. Drachev, V.P.; Chettiar, U.K.; Kildishev, A.V.; Yuan, H.-K.; Cai, W.; Shalaev, V.M. The Ag dielectric function in plasmonic metamaterials. Opt. Express 2008, 16, 1186. [CrossRef] [PubMed]

34. Monti, A.; Bilotti, F.; Toscano, A. Optical cloaking of cylindrical objects by using covers made of core-shell nanoparticles. Opt. Lett. 2011, 36, 4479-4481. [CrossRef] [PubMed]

35. Sivhola, A. Homogenization principles and effect of mixing on dielectric behavior. Photonics Nanostruct. Fundam. Appl. 2013, 11, 364-373. [CrossRef]

36. Danaeifar, M.; Granpayeh, N.; Mortensen, N.A.; Xiao, S. Equivalent conductivity method: Straightforward analytical solution for metasurface-based structures. J. Phys. D Appl. Phys. 2015, 48, 385106. [CrossRef]

37. Tretyakov, S. Analytical Modeling in Applied Electromagnetics; Artech House: Norwood, MA, USA, 2003.

38. Moitra, P.; Slovick, B.A.; Ivan, W.L.; Kravchencko, I.; Briggs, D.P.; Krishnamurthy, S.; Valentine, J. Large-scale all-dielectric metamaterial perfect reflectors. ACS Phot. 2015, 2, 692-698. [CrossRef]

39. Rozanov, K.N. Ultimate thickness to bandwidth ratio of radar absorbers. IEEE Trans. Antennas Propag. 2000, 48, 1230-1234. [CrossRef]

40. Murphy, C.J.; Sau, T.K.; Gole, A.M.; Orendorff, C.J.; Gao, J.; Gou, L.; Hunyadi, S.E.; Li, T. Anisotropic metal nanoparticles: Synthesis, assembly, and optical applications. J. Phys. Chem. B 2005, 109, 13857-13870. [CrossRef] [PubMed]

41. Khodashenas, B.; Ghorbani, H.R. Synthesis of silver nanoparticles with different shapes. Arab. J. Chem. 2015. [CrossRef]

42. Sivhola, A. Dielectric polarization and particle shape effects. J. Nanomater. 2007, 2007, 9. [CrossRef]

(C) 2019 by the authors. Licensee MDPI, Basel, Switzerland. This article is an open access article distributed under the terms and conditions of the Creative Commons Attribution (CC BY) license (http:/ / creativecommons.org/licenses/by/4.0/). 